\title{
Review Article \\ Skin Cancer Knowledge, Attitudes, and Practices among Chinese Population: A Narrative Review
}

\author{
Philip M. Stephens $\mathbb{D}^{1},{ }^{1}$ Brian Martin, ${ }^{1}$ Ghazal Ghafari, ${ }^{1}$ \\ James Luong, ${ }^{1}$ Vinayak K. Nahar $\left(\mathbb{1},{ }^{2}\right.$ Linda Pham, ${ }^{1}$ Jiangxia Luo, ${ }^{3,4}$ \\ Marcelle Savoy, ${ }^{5}$ and Manoj Sharma $\mathbb{1}^{6}$ \\ ${ }^{1}$ DeBusk College of Osteopathic Medicine, Lincoln Memorial University, Harrogate, TN, USA \\ ${ }^{2}$ Center for Animal and Human Health in Appalachia, College of Veterinary Medicine, DeBusk College of Osteopathic Medicine, \\ and School of Mathematics and Sciences, Lincoln Memorial University, Harrogate, TN, USA \\ ${ }^{3}$ Department of English, Gannan Medical University, Ganzhou, Jiangxi, China \\ ${ }^{4}$ Carter and Moyers School of Education, Lincoln Memorial University, Harrogate, TN, USA \\ ${ }^{5}$ Lon and Elizabeth Parr Reed Health Sciences Library, DeBusk College of Osteopathic Medicine, Lincoln Memorial University, \\ Harrogate, TN, USA \\ ${ }^{6}$ Department of Behavioral \& Environmental Health, School of Public Health, Jackson State University, Jackson, MS, USA
}

Correspondence should be addressed to Vinayak K. Nahar; naharvinayak@gmail.com

Received 31 January 2018; Accepted 22 April 2018; Published 4 June 2018

Academic Editor: Gavin P. Robertson

Copyright (C) 2018 Philip M. Stephens et al. This is an open access article distributed under the Creative Commons Attribution License, which permits unrestricted use, distribution, and reproduction in any medium, provided the original work is properly cited.

Skin cancers are becoming a substantial public health problem in China. Fair skin and increased exposure to ultraviolet B (UVB) rays from the sun are among the most substantial risk factors for skin cancer development, thus making the Chinese people vulnerable to this group of diseases. The purpose of this article is to present a narrative review of the knowledge, attitudes, and practices (KAP) related to skin cancers within the Chinese population. A systematic electronic search of MEDLINE (PubMed), CINAHL, ScienceDirect, and Google Scholar databases yielded nine articles that met the inclusion criteria. The review found that although sunscreen application was a commonly used method of skin protection among the general Chinese population, educational interventions enhancing current knowledge and attitudes about the effects of UVB rays on skin from undue sun exposure were limited in many smaller communities of the country. Hence, there is an essential need to design effective, evidencebased educational programs promoting sun protection behaviors in both congregated and sparsely populated areas of China.

\section{Introduction}

Skin cancers, the most commonly diagnosed of all cancers, are typically viewed as ailments affecting primarily Caucasian populations in countries such as Australia, New Zealand, Slovenia, and Norway [1-3]. However, worldwide skin cancer rates have increased over the last three decades [4]. Populations which previously had low skin cancer rates may present the biggest challenge for public health officials due to the lack of established preventative measures. The rise in the incidence of skin cancer is becoming a significant public health problem in China. Between the years of 1990 and 1999, the rate of basal cell carcinoma (BCC) in the nearly doubled from 16.0 to 31.8 per 10,000 new cases, contributing to $60 \%$ of newly diagnosed skin cancers [5]. Squamous cell carcinoma (SCC) is the second most diagnosed type of skin cancer among the Chinese population [5]. Also reflected in the overall number of cutaneous malignancies is the increased mortality rate from melanoma between 1988 and 2007 in the cities of Shanghai and Beijing [6].

The majority of the Chinese population have fair skin along with increased exposure to ultraviolet B (UVB) rays from the sun-creating a heightened risk for the development of skin cancer diseases [7,8]. Specifically, BCC is linked with short-term burning incidents or long-term exposure of the head and neck; SCC is often related to extended 
periods of short- and long-term sun exposure [9]. Thus, skin cancer rate reduction warrants use of sun protective strategies to lower UV absorption. Examples of modifiable behaviors include using sunscreen, limiting direct sun exposure, and wearing protective clothing. Therefore, addressing attitudes and perceptions related to skin cancer within the Chinese population can lead to the initiation and continuation of sun protective behaviors.

Local governments in China distribute treatment expenditures but typically fail to provide money for preventative medicine [10]. Since 2004, the central government has worked to increase cancer prevention funding [10]. As China's rapidly aging population continues to strain its healthcare system financially, preventative measures are of utmost importance due to their cost-saving benefits. The importance of these actions extends beyond the elderly of China's population.

A study conducted on staff and volunteers from seven different Olympic event locations in Beijing found that $79.3 \%$ were aware of the association between UV exposure and skin cancers. Nevertheless, only $49.3 \%$ of participants wore protective clothing, $45.3 \%$ used sunglasses, and $58.8 \%$ applied sunscreen [11]. In another study, sunscreen usage rates were as low as $23.1 \%$ among college-age Chinese residents [12]. These findings suggest that more intervention regarding sun safety should be provided to young Chinese adults. Education is especially important due to a significant risk of SCC from early age exposure in males and lifetime sun exposure in females [9].

This article presents a narrative review on the knowledge, attitudes, and practices (KAP) related to skin cancers within the Chinese population. Based on this framework, recommendations of preventative public health strategies to engage the population have been made.

\section{Methods}

In the initial search, a systematic, computer-based literature search was conducted using MEDLINE (PubMed), Cumulative Index to Nursing and Allied Health Literature (CINAHL), and ScienceDirect. The search was performed in these electronic databases using combinations of the following terms: "China", "Chinese", "skin cancer", "melanoma", "sun protection", "sun behaviors", "knowledge", "attitudes", "beliefs", "perceptions", "sunscreen", "prevention", "practices", and "behaviors". Results of the initial literature search conducted in August 2017 included reviews of abstracts and titles with exclusion of off-topic articles.

Analyses of further studies updated in October 2017 were performed by reviewing reference lists of the articles of interest, along with searches in Google Scholar. The search was not limited by date of publication or language. Both English and Chinese language studies that contained digital and searchable English abstracts and keywords were included.

To the best of the reviewers' knowledge, all manuscripts published in peer-reviewed journals in the selected databases were considered for inclusion in this review. Research studies that measured skin cancer or sun protection related

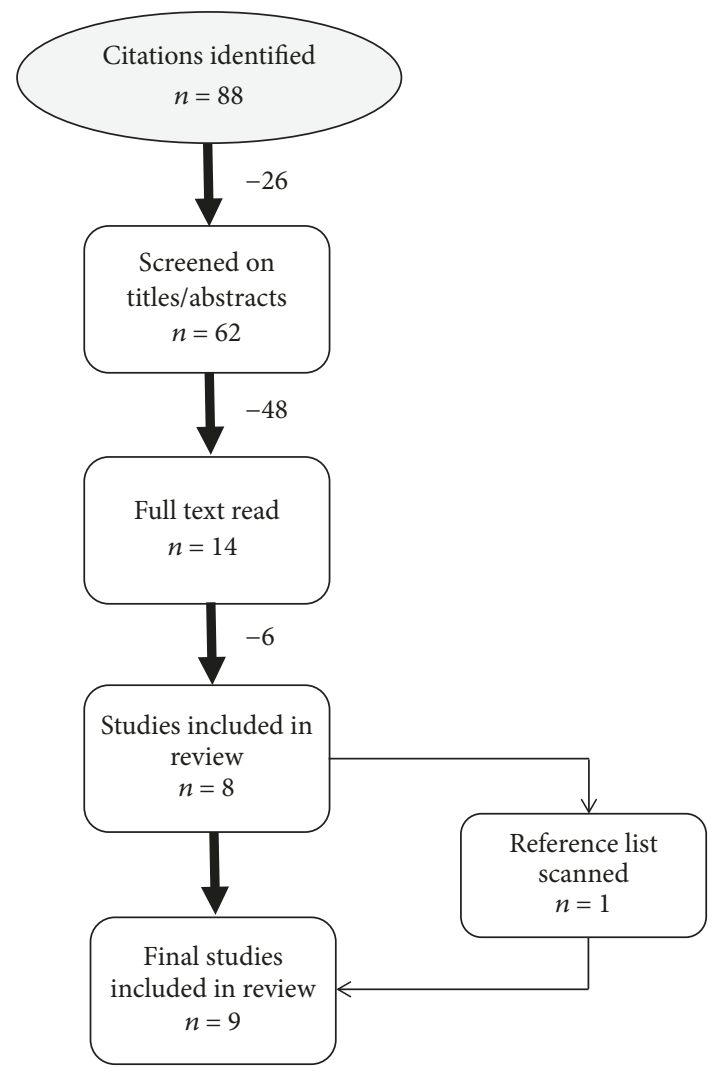

FIGURE 1: Chart of literature review.

knowledge, attitudes, beliefs, and behaviors in China were included. Exclusion criteria for the articles included the following: (1) irrelevant topics to review article aim, (2) articles that focused on treatment of skin cancers instead of preventative practices, and (3) comparable studies conducted on similar groups not indigenous to China. The literature search was conducted by four independent reviewers. Any disagreement regarding inclusion criteria was resolved via discussion until consensus was reached.

\section{Results}

Electronic searches identified a total of 88 citations. After removing duplicates $(n=26)$, the remaining 62 articles were screened based on titles and abstracts. After screening, the remaining 14 articles were read in their entirety to determine inclusion criteria eligibility. In summary, a total of nine articles met the eligibility criteria and were included in the review (Figure 1).

Table 1 provides details of the literature review pertaining to skin cancer knowledge, attitudes, and practices among the Chinese population. The first column displays the senior author of each corresponding article, as well as the date and location of the study. Column two includes the methodologies used for data collection and sample size $(n)$ for each study, along with the gender and age of the participants. Extracted data regarding knowledge, attitudes, and beliefs of the participants are located within the third column. Only preintervention data were extracted and included in the 


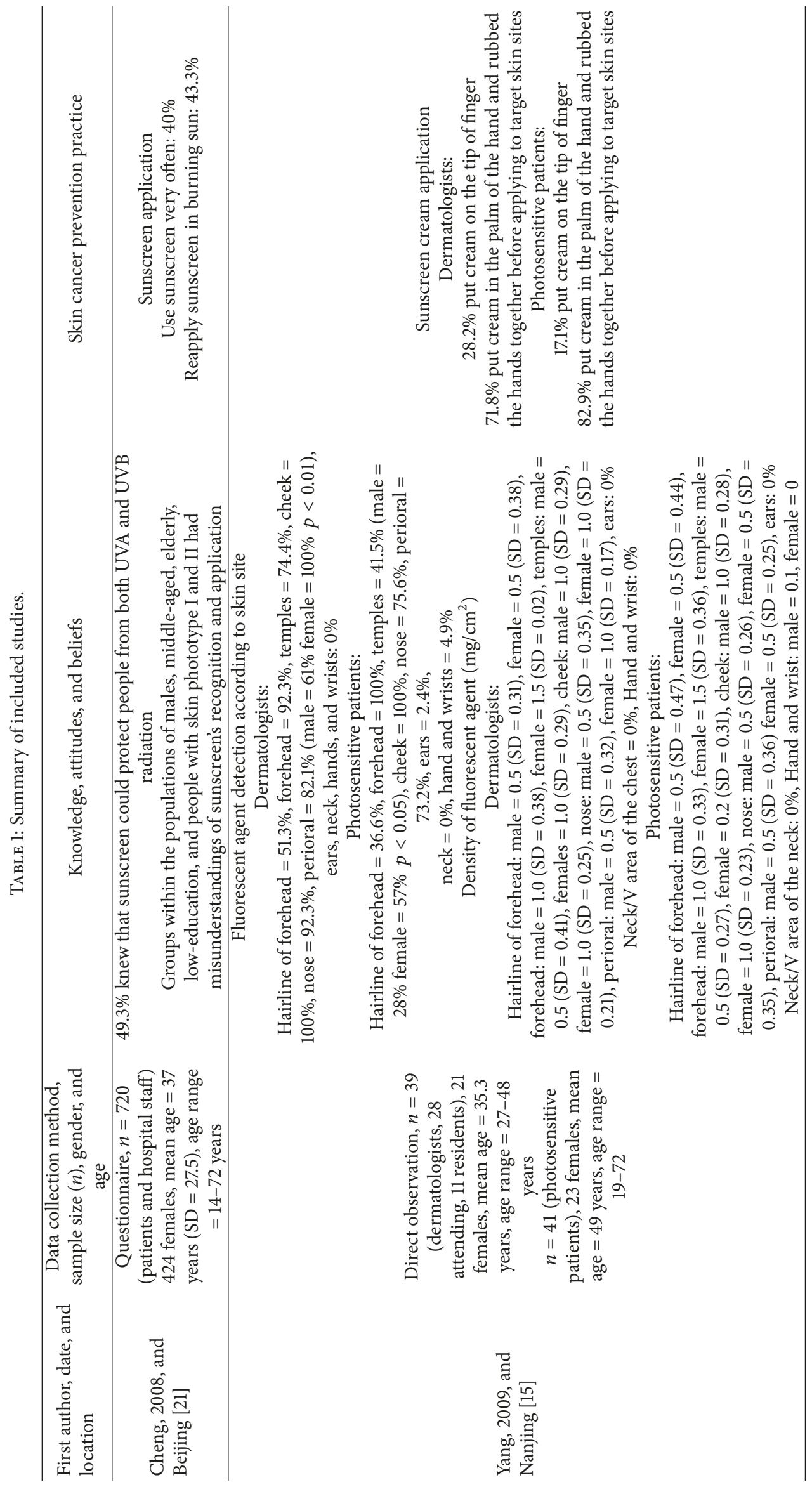




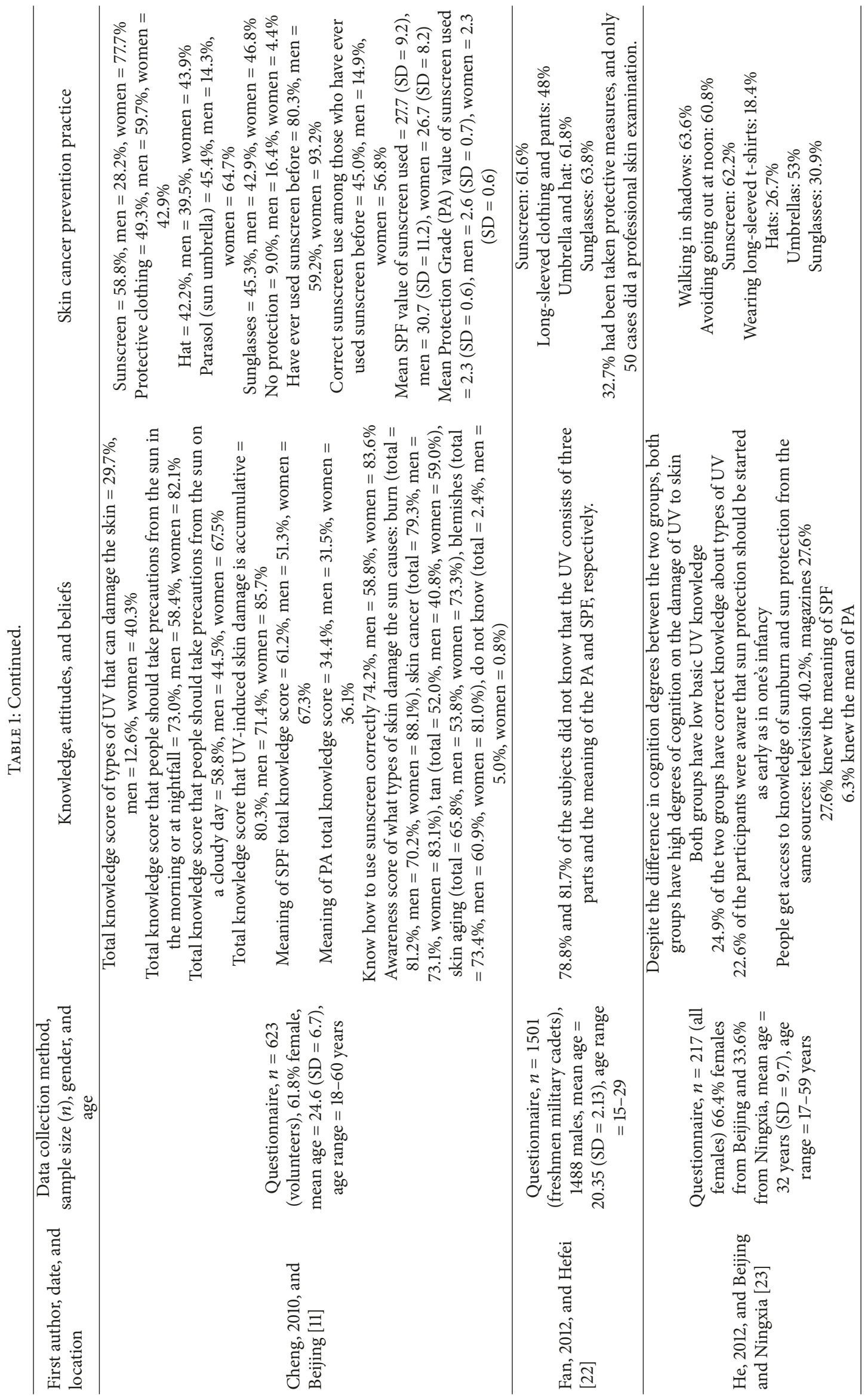




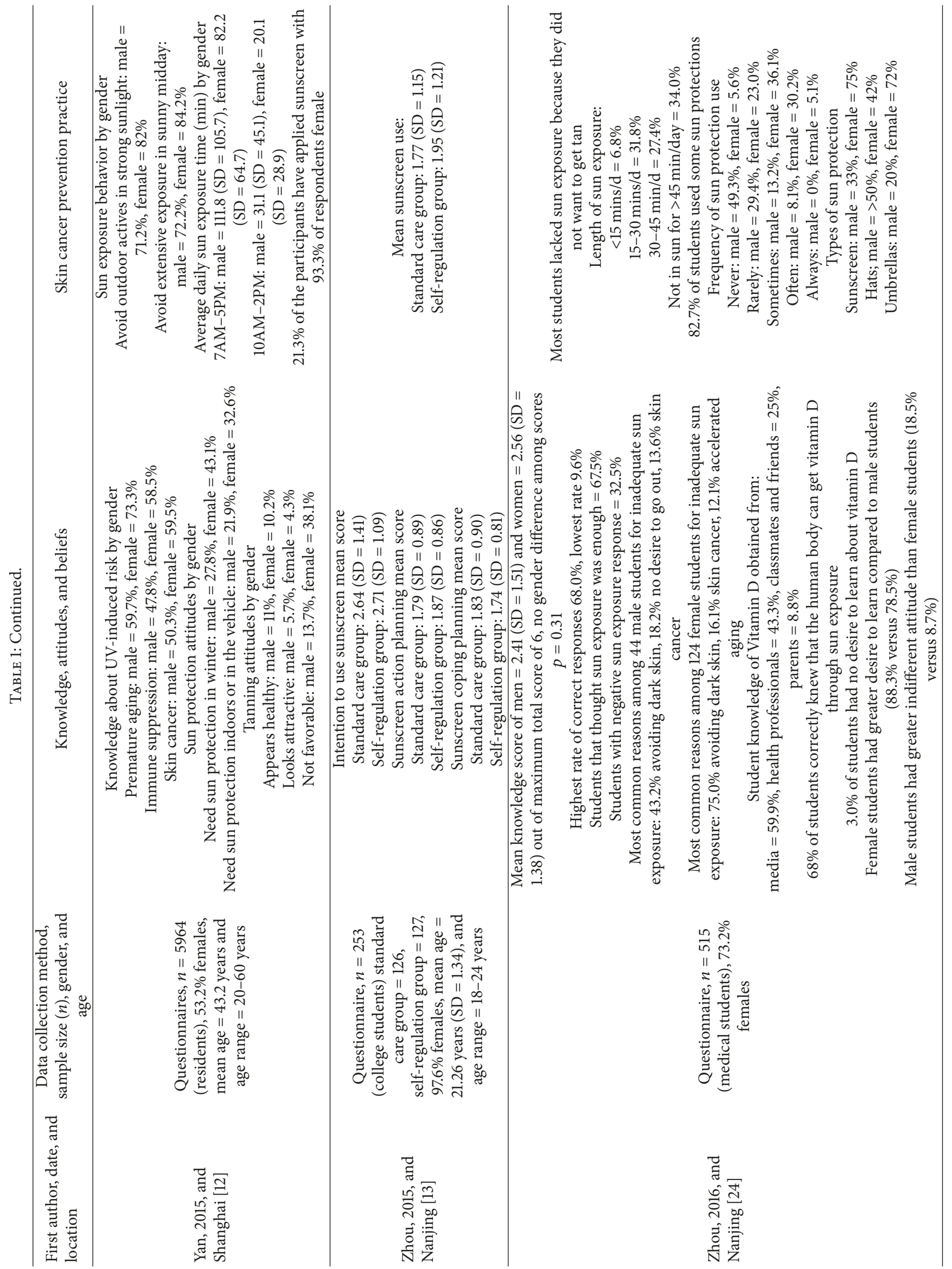




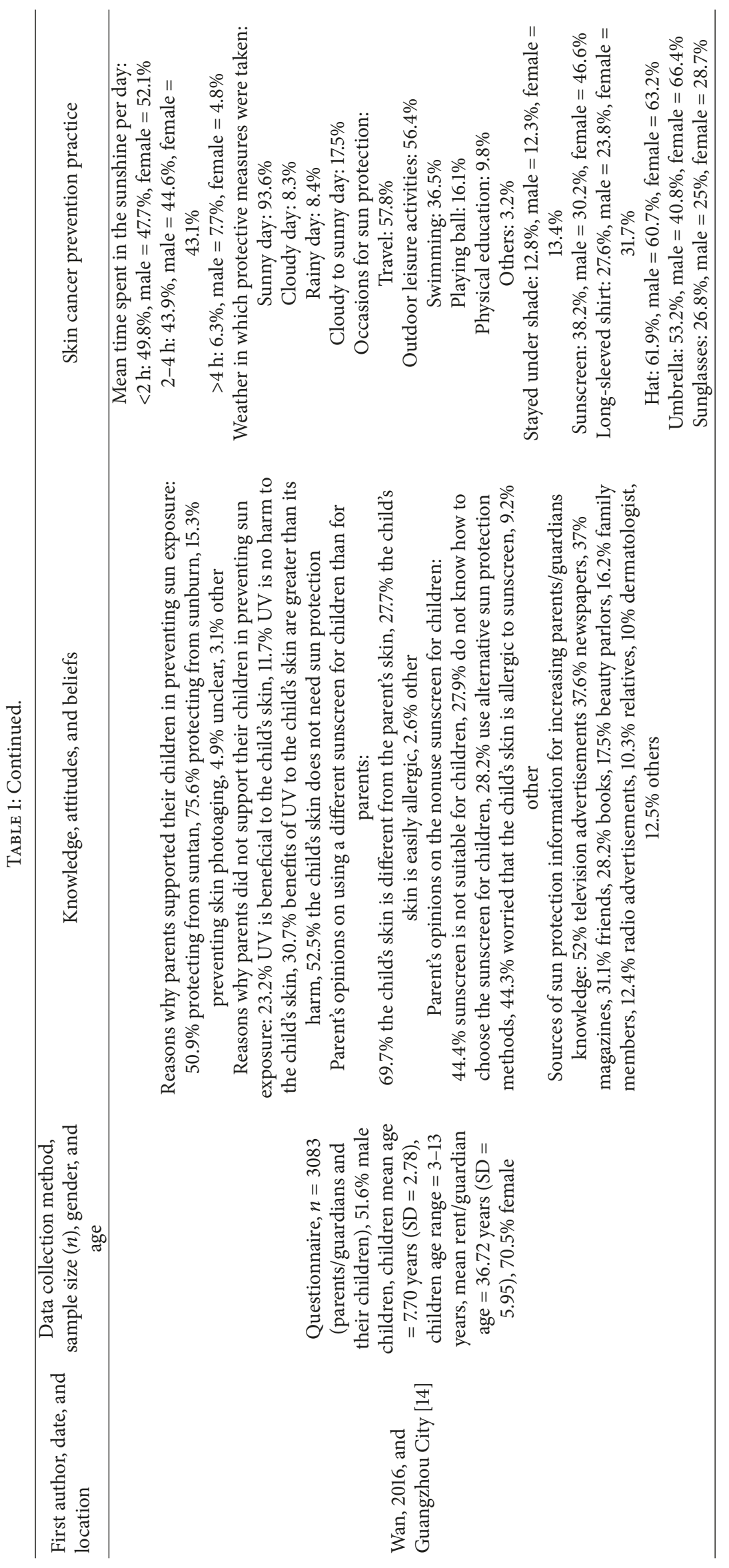


table for studies utilizing experimental design. Prevention practices of participants in each study are described in the last column.

\section{Discussion}

The purpose of this article was to conduct a narrative review to summarize the knowledge, attitudes, and practices (KAP) related to skin cancers within the Chinese population. The Chinese are generally fair-skinned thus making them vulnerable to cutaneous cancers. The other primary modifiable preventative risk factor for skin cancers is exposure to UVB radiation, especially from the sun. The best protective measure, therefore, is limiting exposure to sunlight, particularly during the peak hours between $10 \mathrm{am}$ and $2 \mathrm{pm}$. Other methods suggested to reduce skin cancer incidence include covering the skin with protective clothing-sunglasses, long sleeves, and hats-and the regular application of sunscreens with a sufficiently high sun protection factor (SPF) [8]. Sunscreen use was found to be a popular method of skin protection due to its enhanced affordability in an improving Chinese economy [11]. However, these findings were not in agreement with the results from a survey conducted among 11 communities in Shanghai, where only $21.3 \%$ utilized sunscreen, while the majority of the other participants preferred shade or hats as their primary means of skin protection [12].

While the prevalence of skin cancer is lower in China relative to some of the Western countries [11], BCC and SCC remain common cancers; therefore, skin protection remains important for public health [12]. One possible explanation for the reduced incidence of skin cancer may be that the average complexion among the Chinese populace is not as fair as that found in Western countries. One study found that the majority of the Chinese population is skin type IV on the Fitzpatrick scale, making them amenable to easy tanning and rare burning [12]. Darker-skinned populations have a lower incidence of skin cancer in general but are likely to be diagnosed with more advanced stages of the disease, if it does develop [12]. Another possible explanation may be that, in contrast to Western populations, many Chinese citizens find paler complexions more desirable and are therefore more likely to avoid unnecessary sunlight exposure and tanning [13].

Studies have found educational programs to reduce rates of sunburn and skin aging in the general population [11]. However, based on this review we found that such initiatives are rather few in China, with the effects of UVB being largely ignored [12]. Furthermore, knowledge and attitudes pertaining to UVB radiation have been found to be somewhat deficient among many Chinese communities. Among men and women, one study found that only $55.2 \%$ knew that UV radiation causes skin cancer [12]. Similar to Western populations, women scored higher on skin protective knowledge tests than men [11, 12]. Men were found to be less likely to care for their skin regularly compared to women, with many men expressing that sunscreen and umbrella usage are women's activities. Men were also less willing to participate in educational programs about skin protection [11]. Additional efforts should be made to improve the population's perspective, particularly men, on skin cancer prevention and to increase their involvement.

Sun exposure during childhood has been correlated with skin cancer development later in life [14]. The percentage of children with a history of experiencing a sunburn increases as age increases. Therefore, it is imperative for educational programs to be introduced at an early age before multiple sunburns have been manifested. Interestingly, a large percentage $(93.6 \%)$ of parents reported utilizing sunscreen for skin protection on their children (including 38.2\% who are less likely to utilize the same methods for themselves), citing sunburn prevention as the main reason [14]. Involving parents in their children's educational program presents a possible avenue for improving adult attitudes as well.

The appropriate application of sunscreens is another area of concern. Frequently, inadequate amounts of sunscreens were found to be used in addition to inconsistent methods of application, i.e., some areas of the body received more sunscreen, leaving other areas at risk. An application density of $2 \mathrm{mg} / \mathrm{cm}^{2}$ has been found to be most appropriate to receive the full SPF of the sunscreen [15]. Parents who did not use sunscreens on their children were fearful either that the products were unhealthy or that they would cause an allergic reaction, but among those who did use this preventative measure, nearly half did not follow the recommended reapplication rate of every 2-3 hours or used a product strength that was often less than 30 SPF [14].

Before introducing UVB awareness and skin protection programs to the Chinese population, modifications should be made to assure cultural and ethical appropriateness. One such concern is the risk of vitamin D deficiency, which Asian adults face due to their complexion [16]. In terms of disseminating knowledge and awareness to the public at large, television advertisements were selected by parents as an important medium for sun protection information (52\% agreement) [14]. This finding suggests that the Chinese population is receiving a substantial portion of its medical information from nonmedical sources. Mass media campaigns, therefore, should attempt to involve medical professionals in their creation to improve the broad dissemination of effective skin protective strategies.

Knowledge alone has not been found to be a strong predictor of behavioral change [13]. The level of health literacy was a significant factor in the relationship between knowledge and behavior. For example, in the context of vitamin $\mathrm{D}$ absorption and sun exposure, individuals with high health literacy were more likely to discern appropriate amounts of sun exposure to maximize the amount of vitamin D absorption; unfortunately, poor attitudes counteracted the benefits of health literacy [17]. This suggests that any educational initiative should not only focus on increasing knowledge and health literacy, but also on improving societal attitudes, which insure the initiation and continuation of healthy behavioral changes related to skin protection and sun exposure. Thus, evidence-based theoretical approaches need to be instituted to foster effectual behavior change [18]. In present day context, fourth generation behavior changes that utilize multiple theories are in vogue [19]. One such utilization is through the multi-theory model (MTM) of 
health behavior change that breaks down behavior change into initiation and sustenance [20]. For initiation of sun protection behaviors attitudes that underscore advantages over disadvantages of such behavior change and behavioral confidence to use sun protection behaviors along with the changes in physical environment that support sun protection are important. In order to sustain sun protective behaviors, one needs to promote conversion of emotions toward goals for adhering to and continually practicing these behaviors, as well as mobilizing changes in social environment.

\section{Limitations}

There are limitations in the construct and the included studies of this narrative review. Some of the studies are susceptible to measurement biases due to self-reported data collection methods. Additionally, the data collected via questionnaires are subject to the recall bias. Although some of the studies were in Chinese with abstracts in English, the search was conducted solely using English language accessible electronic databases; therefore it is possible that some relevant articles were missed. Furthermore, we omitted searches within the grey literature. Finally, caution should be exercised when making generalizations of findings to the Chinese population since this review included only nine studies from six locations.

\section{Conclusions}

The findings of this study indicate a need for increasing awareness and knowledge among the Chinese population about skin cancer risk factors and institute effectual changes and interventions that promote sun protection behaviors. More research is needed with this population to gain a better understanding of attitudes and beliefs and how they could be adjusted into meaningful skin cancer prevention practices. Future studies must attempt to develop evidencebased theoretical interventions to help individuals to initiate and sustain sun protection behaviors in order to decrease their future skin cancer risk. Finally, research should take into consideration behavior change differences between gender, age, and socioeconomic status with regard to sun protection behaviors.

\section{Conflicts of Interest}

The authors declare that they have no conflicts of interest.

\section{References}

[1] F. Xiang, R. Lucas, S. Hales, and R. Neale, "Incidence of nonmelanoma skin cancer in relation to ambient UV radiation in white populations, 1978-2012: empirical relationships," JAMA Dermatology, vol. 150, no. 10, pp. 1063-1071, 2014.

[2] J. Ferlay, E. Steliarova-Foucher, J. Lortet-Tieulent et al., "Cancer incidence and mortality patterns in Europe: estimates for 40 countries in 2012," European Journal of Cancer, vol. 49, no. 6 , pp. 1374-1403, 2013.

[3] A. Lomas, J. Leonardi-Bee, and F. Bath-Hextall, "A systematic review of worldwide incidence of nonmelanoma skin cancer,"
British Journal of Dermatology, vol. 166, no. 5, pp. 1069-1080, 2012.

[4] J. Yu, X. Luo, H. Huang, Z. Zhai, Z. Shen, and H. Lin, "Clinical characteristics of malignant melanoma in southwest China: A Single-Center Series of 82 consecutive cases and a metaAnalysis of 958 reported cases," PLoS ONE, vol. 11, no. 11, Article ID e0165591, 2016.

[5] S. Y. Cheng, N. M. Luk, and L. Y. Chong, "Special features of non-melanoma skin cancer in Hong Kong Chinese patients: 10year retrospective study," Hong Kong medical journal, vol. 7, no. 1, pp. 22-28, 2001.

[6] H. M. Zeng, S. W. Zhang, R. S. Zheng et al., "Analysis of incidence and mortality of cutaneous melanoma from 2003 to 2007 in China," China Cancer, vol. 3, pp. 183-189, 2012.

[7] M. A. Linares, A. Zakaria, and P. Nizran, "Skin Cancer," Primary Care-Clinics in Office Practice, vol. 42, no. 4, pp. 645-659, 2015.

[8] Skin Cancer Facts \& Statistics, "Skin Cancer Foundation website," http://www.skincancer.org/skin-cancer-information/ skin-cancer-facts\#general.

[9] C. Pelucchi, A. Di Landro, L. Naldi, and C. La Vecchia, "Risk factors for histological types and anatomic sites of cutaneous basal-cell carcinoma: An Italian case-control study," Journal of Investigative Dermatology, vol. 127, no. 4, pp. 935-944, 2007.

[10] X. Ma, C. Lin, and W. Zhen, "Cancer care in China: A general review," Biomedical Imaging and Intervention Journal, vol. 4, no. 3, 2008 .

[11] S. Cheng, S. Lian, Y. Hao et al., "Sun-exposure knowledge and protection behavior in a North Chinese population: a questionnaire-based study," Photodermatology, Photoimmunology \& Photomedicine, vol. 26, pp. 177-181, 2010.

[12] S. Yan, F. Xu, C. Yang et al., "Demographic Differences in Sun Protection Beliefs and Behavior: A Community-Based Study in Shanghai, China," International Journal of Environmental Research and Public Health, vol. 12, no. 3, pp. 3232-3245, 2015.

[13] G. Zhou, L. Zhang, N. Knoll, and R. Schwarzer, "Facilitating Sunscreen Use Among Chinese Young Adults: Less-Motivated Persons Benefit from a Planning Intervention," International Journal of Behavioral Medicine, vol. 22, no. 4, pp. 443-451, 2015.

[14] M. Wan, R. Hu, Y. Li et al., "Attitudes, Beliefs, and Measures Taken by Parents to Protect Their Children from the Sun in Guangzhou City, China," Photochemistry and Photobiology, pp. 753-759, 2016.

[15] H. P. Yang, K. Chen, M. Ju, B. Z. Chang, L. Y. Wang, and H. Gu, "A study of the way in which dermatologists and photosensitive patients apply sunscreen in China," Photodermatology, Photoimmunology \& Photomedicine, vol. 25, no. 5, pp. 245-249, 2009.

[16] Q. Gao, G. Liu, and Y. Liu, "Knowledge, attitude and practice regarding solar ultraviolet exposure among medical university students in Northeast China," Journal of Photochemistry and Photobiology B: Biology, vol. 140, pp. 14-19, 2014.

[17] A. Y. M. Leung, M. K. T. Cheung, and I. Chi, "Supplementing vitamin D through sunlight: Associating health literacy with sunlight exposure behavior," Archives of Gerontology and Geriatrics, vol. 60, no. 1, pp. 134-141, 2015.

[18] M. Sharma, Theoretical Foundations of Health Education and Health Promotion, Jones and Bartlett Learning, Burlington, MA, USA, 2017.

[19] M. Sharma, "Trends and prospects in public health education: A commentary," [Editorial]. Social Behavior Research \& Health, vol. 1, no. 2, pp. 67-72, 2017. 
[20] M. Sharma, "Multi-theory model (MTM) for health behavior change," WebmedCentral Behaviour, vol. 6, no. 9, Article ID WMC004982, 2015.

[21] S. W. Cheng, F. Liu, M. Cao et al., "Knowledge and the Use of about Sunscreen Among Chinese Population in Beijing," The Chinese Journal of Dermatovenereology, vol. 22, no. 9, pp. 566569, 2008.

[22] D. Z. Fan, S. Liu, T. Yang, J. L. Wand, K. Ye, and F. M. Pan, "The Investigation and Analysis on the Awareness of Sun Protection Knowledge and the Use of Sunscreen Among Cadets in Hefe," China Medical Abstract of Dermatology, pp. 6-9, 2012.

[23] R. He, "A questionnaire-based study on sun-exposure knowledge and protection behavior in women from North China," Ningxia Medical Journal, pp. 12-031, 2012.

[24] M. Zhou, W. Zhuang, Y. Yuan, Z. Li, and Y. Cai, "Investigation on Vitamin D knowledge, attitude and practice of university students in Nanjing, China," Public Health Nutrition, vol. 19, no. 1, pp. 78-82, 2016. 


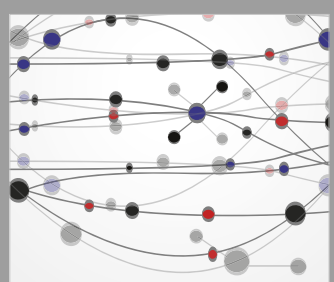

The Scientific World Journal
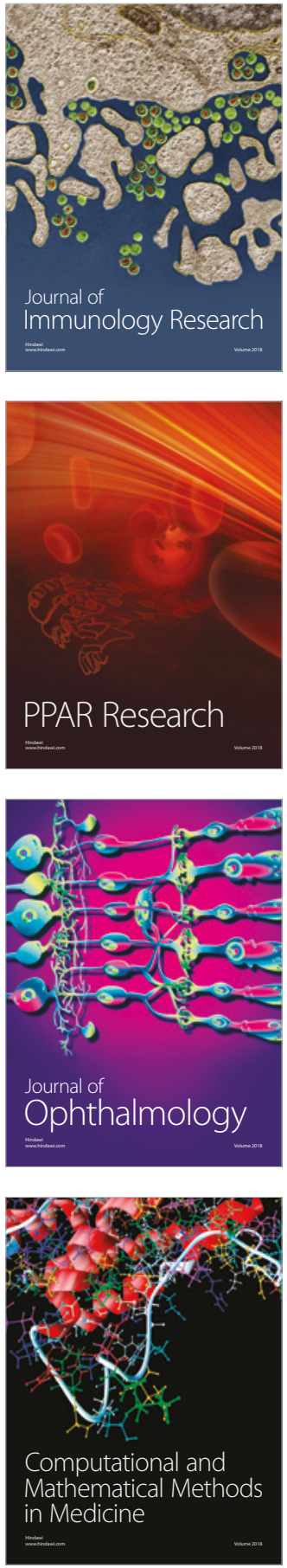

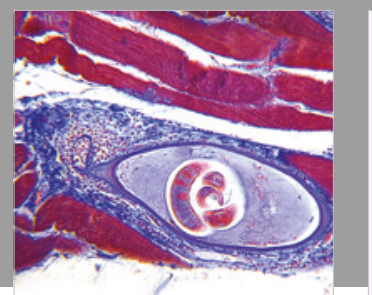

Gastroenterology Research and Practice

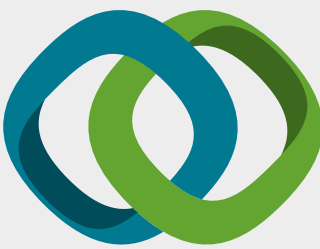

\section{Hindawi}

Submit your manuscripts at

www.hindawi.com
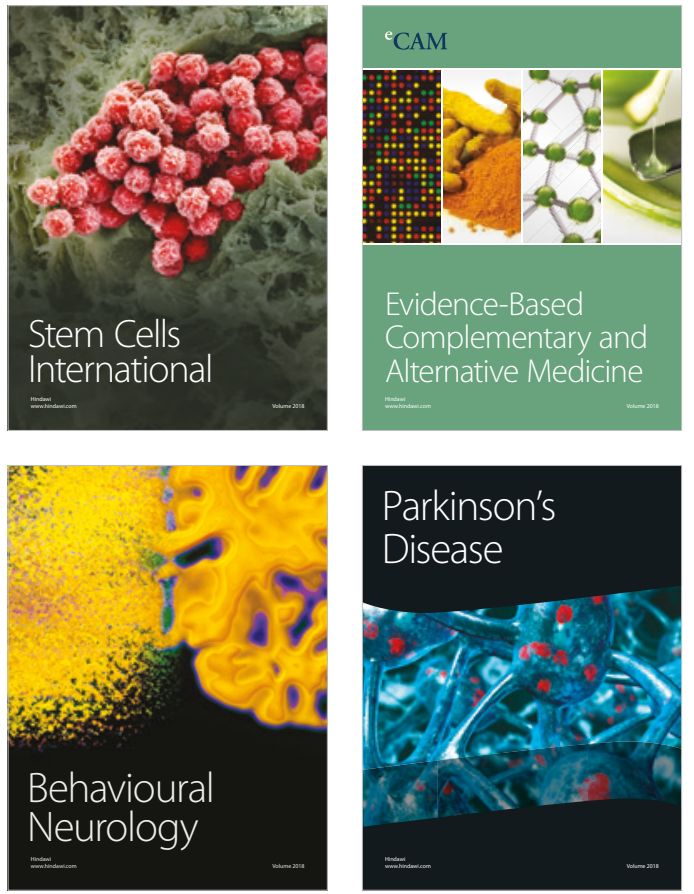

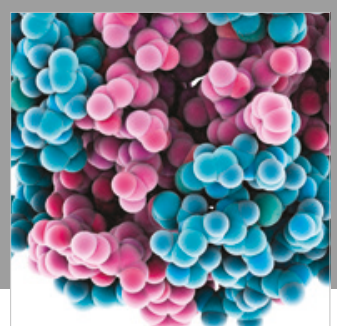

ournal of

Diabetes Research

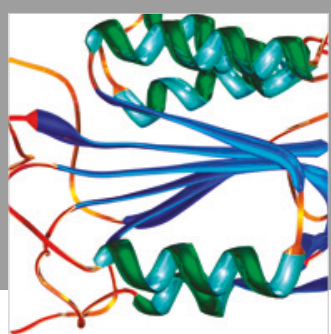

Disease Markers
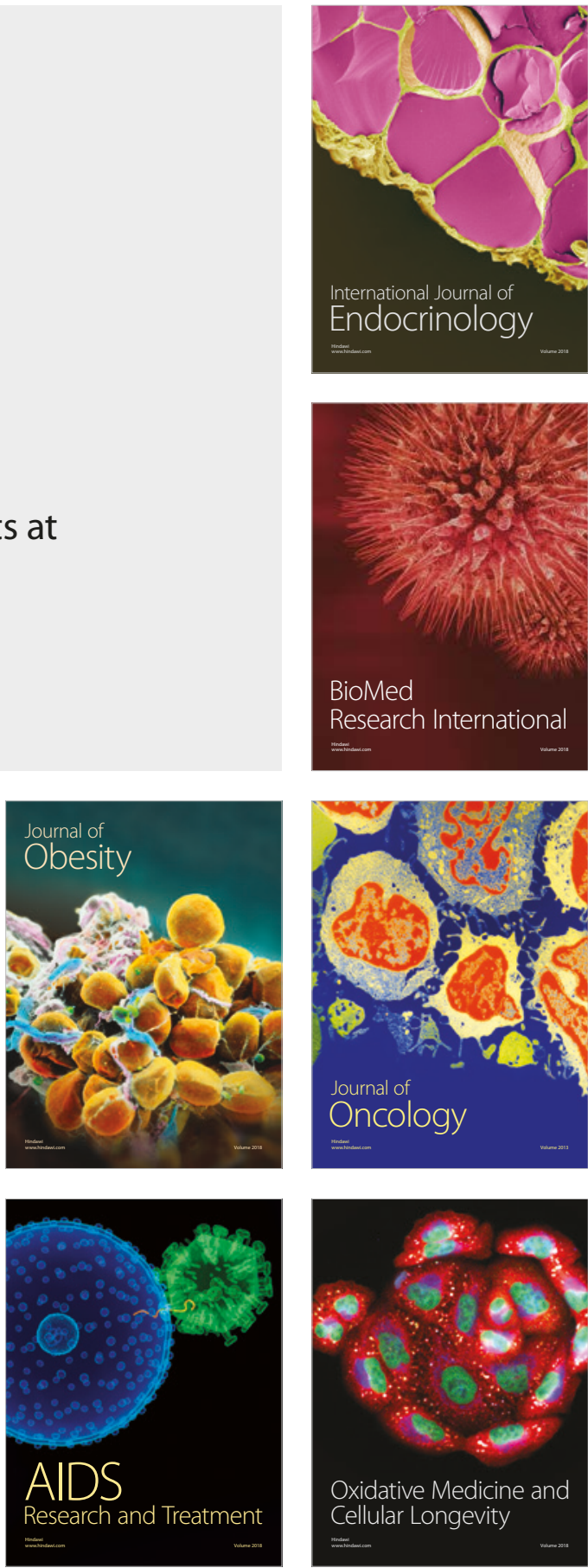